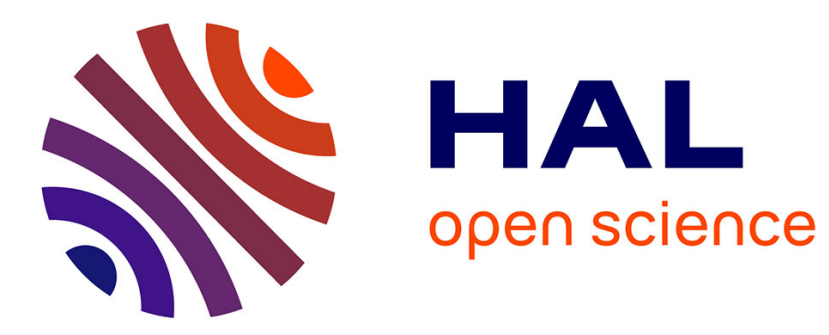

\title{
Some results on the convergence of transfer function expansions on the Laguerre series
}

\author{
Rachid Malti, Didier Maquin, José Ragot
}

\section{To cite this version:}

Rachid Malti, Didier Maquin, José Ragot. Some results on the convergence of transfer function expansions on the Laguerre series. 5th European Control Conference, ECC'99, Aug 1999, Karlsruhe, Germany. pp.CDROM. hal-00283923

\section{HAL Id: hal-00283923 \\ https://hal.science/hal-00283923}

Submitted on 31 May 2008

HAL is a multi-disciplinary open access archive for the deposit and dissemination of scientific research documents, whether they are published or not. The documents may come from teaching and research institutions in France or abroad, or from public or private research centers.
L'archive ouverte pluridisciplinaire HAL, est destinée au dépôt et à la diffusion de documents scientifiques de niveau recherche, publiés ou non, émanant des établissements d'enseignement et de recherche français ou étrangers, des laboratoires publics ou privés. 


\title{
SOME RESULTS ON THE CONVERGENCE OF TRANSFER FUNCTION EXPANSION ON LAGUERRE SERIES
}

\author{
Rachid MALTI, Didier MAQUIN, José RAGOT \\ Centre de Recherche en Automatique de Nancy \\ Institut National Polytechnique de Lorraine \\ 2, Avenue de la Forêt de Haye - 54516 Vandoeuvre-lès-Nancy \\ Tél. (33) $0383595684 \quad$ Fax (33) 0383595644
}

\begin{abstract}
When a transfer function is expanded on the basis of Laguerre filters, the question of how well does the expansion converge arises frequently. Beyond this problem, the convergence domain of the Laguerre series must be determined in the $s$-plane, as is usually done for the Laplace transform of time-domain functions. In the usual approach, this analysis is made in two complementary stages: first of all, the convergence conditions of Fourier (also called Laguerre or Laguerre-Fourier) coefficients is determined and then, based on the assumption that these coefficients are convergent, a worst-case-study is carried out to determine the convergence domain of the Laguerre series. A novel approach is proposed in this paper which drops away the coupling between the convergence of the Fourier coefficients and the convergence of the Laguerre series. Thus, necessary and sufficient conditions for Laguerre series convergence are computed. Laguerre functions are considered in their general definition : orthogonal w.r.t. an exponential weight function.
\end{abstract}

\section{Introduction}

The use of orthogonal bases functions for system and signal representations is very common in different fields of signal processing and control engineering. Among the most commonly used bases, the one which is obtained from Laguerre polynomials offers particular interest, especially in the field of automatic control [Bélanger et al., 1994], [Agamemmomi et al., 1992]. There remains, however, few points still under discussion such as the optimal choice of the Laguerre pole (see for instance [Wang et al., 1994] and [Malti et al., 1998b]), the determination of the truncation order and the influence of noisy measurements in the resulting approximation.

Usually, when the Laplace transform of a time function is computed, its convergence domain in the complex $s$-plane is systematically determined. In this paper we are interested in defining the convergence domain (in the $s$-plane) for Laguerre series expansions. Compared to the existing work, the usually computed convergence domain is extended. In other words, necessary and sufficient conditions are determined.

The paper is organized in the following manner. After a brief reminder on transfer function expansions on Laguerre series, the usual approach for computing the convergence domain is presented. It is based on the study of the convergence of Fourier coefficients (presented in the second part of the paper) and then according to the previous result the domain of convergence of the Laguerre series expansion is determined (third part). Then, in the fourth section, a novel approach is formulated. It drops away the coupling between the convergence of the Fourier coefficients and the convergence of the Laguerre series. The fifth part is devoted to some academic examples and at last, an application on the bilateral expansion of non-causal signals is analyzed, by analogy to the bilateral Laplace transform.

\section{Decomposition of a first order transfer function}

The continuous time Laguerre functions defined by:

$$
l_{n}(t)=\frac{e^{-(\alpha-\gamma) t}}{n !} \frac{d^{n}\left(t^{n} e^{-\gamma t}\right)}{d t^{n}}
$$

have the following Laplace transform:

$$
L_{n}(s)=\frac{(s-\gamma+\alpha)^{n}}{(s+\alpha)^{n+1}}
$$

also known as Laguerre filters. They are orthonormal w.r.t. the weight function:

$$
p(t)=\gamma e^{-(\gamma-2 \alpha) t}
$$

since they satisfy the following equation:

$$
\left\langle L_{n}(t), L_{m}(t)\right\rangle_{p(t)}=\int_{0}^{\infty} L_{n}(t) L_{m}(t) p(t) d t=\delta_{n m}
$$

where $\langle., .\rangle_{p(t)}$ defines the scalar product w.r.t. the weight function $p(t)$ (see Szegö, 1931 for more details).

Moreover, they form a complete orthogonal set in $\mathrm{L}^{2}[0, \infty[$. Actually, the mostly known Laguerre functions, in control engineering literature corresponds to the choice of $\gamma=2 \alpha$, where the weight function is reduced to a constant factor $p(t)=2 \alpha$ which square root is injected back in the definition of Laguerre functions (1), in order to satisfy to the normality property w.r.t. a unity weight.

\section{Remarks}

1. Laguerre functions are a priori defined for any values of $\alpha$ and $\gamma$. However, in order to ensure their convergence, as $t$ tends to infinity, and by the way obtain stable Laguerre filters, the choice of $\alpha$ is restricted to the following condition :

$$
\alpha>0
$$

2. Moreover, the Laguerre functions are orthogonal iff (see [Malti et al., 1998a] for the complete proof):

$$
\gamma>0
$$

3. On the other hand, the Laplace transform of Laguerre filters exists iff:

$$
\mathfrak{R}(s)>-\alpha
$$


The Fourier coefficients are computed by minimizing the weighted least squares criterion:

$$
\Phi=\int_{0}^{\infty}(f(t)-\tilde{f}(t))^{2} p(t) d t
$$

where $: \tilde{F}(s)=\sum_{n=0}^{\infty} a_{n} \frac{(s-\gamma+\alpha)^{n}}{(s+\alpha)^{n+1}}$

and $\mathcal{L}(f(t))=\tilde{F}(s)$

Equation (3) corresponds to the weighted quadratic norm of the approximation error, according to the definition of the scalar product (2):

$$
\Phi=\|f(t)-\tilde{f}(t)\|_{p(t)}^{2}
$$

Minimizing the least squares criterion (3) and taking advantage of the orthogonality of Laguerre functions, the Fourier coefficients are obtained by performing the following integration in the time domain:

$$
a_{n}=\int_{0}^{\infty} f(t) l_{n}(t) e^{-(\gamma-2 \alpha) t} d t
$$

which reciprocal in the frequency domain, obtained by Parseval's theorem, leads to the computation of the residuals as indicated below:

$$
a_{n}=\left.\frac{\gamma}{n !} \frac{d^{n}}{d s^{n}}\left(F(s)(s+\alpha)^{n}\right)\right|_{s=\gamma-\alpha}
$$

For example, for the transfer function $F(s)=\frac{A_{i}}{s+s_{i}}$, (where $s_{i}$ is real) the Fourier coefficients are given by:

$$
a_{n}=\gamma A_{i} \frac{\left(s_{i}-\alpha\right)^{n}}{\left(s_{i}-\alpha+\gamma\right)^{n+1}}
$$

\section{Remarks}

1. $F(s)$, the Laplace transform of $f(t)=A_{i} e^{-s_{i} t}$ exists, if and only if $s>\sigma_{f}, \sigma_{f}$ being the abscissa of convergence (in the previous example $\sigma_{f}=-s_{i}$ ).

2. The generalization to higher order transfer functions with multiple poles is straightforward. For the sake of simplicity and without loss of generality, the coming examples will be considered for the first order transfer functions :

$$
F(s)=\frac{A_{i}}{s-s_{i}}
$$

As shown in (6), the Fourier coefficients of the Laguerre expansion depend on the Laguerre pole $\alpha$ and the parameter $\gamma$. Hence, it is important to make a case-study depending on $\alpha$ and $\gamma$ while searching for the convergence conditions of a first order transfer function having a single pole $s_{i}$.

\section{Remark}

The infinite series (4) is often truncated at an order $(N+1)$ and the following approximation of $F(s)$ is frequently made:

$$
\tilde{F}_{N}(s)=\sum_{n=0}^{N+1} a_{n} \frac{(s-\gamma+\alpha)^{n}}{(s+\alpha)^{n+1}}
$$

Thanks to the orthogonality, the computation of the Fourier coefficients, using (6) when making this truncation remains unchanged. 囚

\section{Convergence of the Fourier coefficients}

It appears from (4) that the Fourier coefficients, $a_{n}$ evolve as a complex geometrical series which convergence to zero as $N$ tends to infinity is satisfied if the norm of its ratio is less than one:

$$
\left|\frac{s_{i}-\alpha}{s_{i}-\alpha+\gamma}\right|<1
$$

The geometrical interpretation of this condition in the $s$-plane can be given after defining:

$S_{i}$ as the image of the pole $s_{i}$

$A$ as the image of $\alpha$

$B$ as the image of $\alpha-\gamma$.

Note that $A S_{i}$ and $B S_{i}$ are the images of $\left(s_{i}-\alpha\right)$ and $\left(s_{i}-\alpha-\gamma\right)$ respectively. Hence, the inequality (8) means that $\overline{A S_{i}} / \overline{B S_{i}}<1$. In other words, $S_{i}$ must be on the LHS of the axis of abscissa $(-\alpha+\gamma / 2)$.

From an algebraic point of view, it is easy to check that the pole $s_{i}$ of the transfer function must satisfy the following inequality :

$$
\Re\left(-s_{i}\right)<-\alpha+\frac{\gamma}{2}
$$

The convergence condition expressed by (9), can be generalized to higher order transfer functions. Indeed, if multiple poles are present, $s_{i}$ is substituted by the dominating pole (closest to the imaginary axis).

\section{Convergence of the Laguerre series expansion}

In this section the transfer function $F(s)$ defined in (7) is compared to its expansion. Using (4) and (6), $\tilde{F}(s)$ can be written as:

$$
\tilde{F}(s)=\gamma A_{i} \sum_{n=0}^{\infty} \frac{\left(s_{i}-\alpha\right)^{n}}{\left(s_{i}-\alpha+\gamma\right)^{n+1}} \frac{(s-\gamma+\alpha)^{n}}{(s+\alpha)^{n+1}}
$$

It represents an infinite sum of a complex geometrical series which general term is:

$$
u_{n}=\gamma A_{i} \frac{\left(s_{i}-\alpha\right)^{n}}{\left(s_{i}-\alpha+\gamma\right)^{n+1}} \frac{(s-\gamma+\alpha)^{n}}{(s+\alpha)^{n+1}}
$$

Moreover, this series is convergent if the norm of its ratio is less than one, i.e. if:

$$
\left|\frac{-s_{i}+\alpha}{-s_{i}+\alpha-\gamma} \frac{s-\gamma+\alpha}{s+\alpha}\right|<1
$$

Usually, condition (11) is analyzed in the worst-case study by assuming that the Fourier coefficients are convergent as $n$ tends to $\infty$ (condition (8) is satisfied). This (11) is reduced to:

$$
\left|\frac{s-\gamma+\alpha}{s+\alpha}\right|<1
$$


Again from a geometrical point of view, the point $M$ which affixe is $s$ must be on the RHS of the vertical axis of abscissa : $(-\alpha+\gamma / 2)$. From an algebraic point of view, (12) is simplified to:

$$
\mathfrak{R}(s)>-\alpha+\frac{\gamma}{2}
$$

\section{Novel proposition (less restrictive)}

We wish to examine again the inequality (11). The proposed approach is intrinsic, i.e. it does not take into account the convergence of the Laguerre coefficients $a_{n}$. The proposition formulated in this paragraph is original and gives a necessary and sufficient conditions for the convergence of the geometrical series (10). Assuming that $M$ is the image of $s$ with coordinates $(x, y)$, then the condition (11) can directly be written as:

$$
\frac{\overline{A S_{i}}}{\overline{B S_{i}}} \frac{\overline{B M}}{\overline{A M}}<1
$$

Hence, it can be shown that the locus of the points $M$ satisfying the condition $\overline{A S_{i} \overline{B M}}=\overline{B S_{i} \overline{A M}}$ is a circle. On the other hand, from an algebraic point of view, (11) is equivalent to :

$$
\left(\delta_{i}^{2}-1\right)\left(\left(x+a_{i}\right)^{2}+y^{2}-r_{i}^{2}\right)<0
$$

where: $x=\mathfrak{R}\{s\}, y=\mathfrak{I}\{s\}$

$$
\begin{aligned}
& \delta_{i}=\left|\frac{-s_{i}+\alpha}{-s_{i}+\alpha-\gamma}\right|=\frac{\overline{A S}_{i}}{\overline{B S}_{i}}, a_{i}=\alpha-\frac{\gamma \delta_{i}^{2}}{\delta_{i}^{2}-1} \\
& r_{i}=\left|\frac{\gamma \delta_{i}}{\delta_{i}^{2}-1}\right|
\end{aligned}
$$

Thus, the inequality (14a) gives the locus of the points $M$ which ensure the convergence of the Laguerre series expansion of $F(s)$. According to the sign of $\left(\delta_{i}^{2}-1\right)$ this locus will be either inside or outside the circle which equation is:

$$
\left(x+a_{i}\right)^{2}+y^{2}-r_{i}^{2}=0
$$

Moreover, according to the Laguerre parameters $\alpha, \gamma$ and the pole $s_{i}$ of $F(s)$, the convergence domain varies in the shape and the position. A crucial point, in this analysis, concerns the existence of an intersection of the convergence domain with the imaginary axis. If it is the case, the convergence of the decomposition in the frequency domain is restricted to some frequencies only. However, if the imaginary axis is not included in the convergence domain, the interest of the Laguerre series expansion is considerably limited, because the Laguerre series is divergent for all frequencies.

One should keep in mind that the abscissa of convergence, of the transfer function must be taken into account:

$$
x>-s_{i}
$$

\section{Example 1}

The domain of convergence of the transfer function $F(s)=\frac{1}{s+3}$ is colored in gray in figure (1). The RHS figure corresponds to the case $\alpha=2$ and $\gamma=3$, whereas the LHS figure corresponds to the case $\alpha=2$ and $\gamma=8$. Note that for both cases $\delta_{i}<1$. The vertical axis at $-s_{i}$ defines the Laplace transform convergence abscissa of $F(s)$. Notice that all the imaginary axis belongs to the convergence domain of the Laguerre series expansion. This ensures a good frequency approximation with a finite number of Laguerre functions.

According to the worst-case study detailed in sections 3 and 4 the convergence domain is located in this example at the RHS of the vertical axis $(-\alpha+\gamma / 2)$.

In the first case this axis is located at -0.5 , whereas in the second one it is located at 2 . The worst-case study gives very restrictive conditions, especially in the latter case where a wrong conclusion may be drawn, since the imaginary axis does not belong to the convergence domain which is shown to be false according to the less restrictive study.

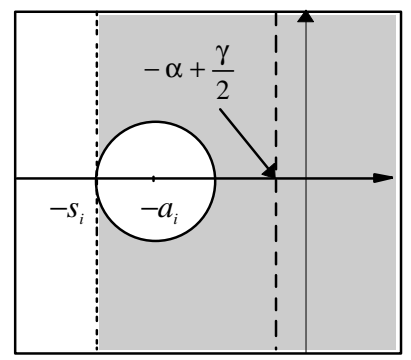

(a)

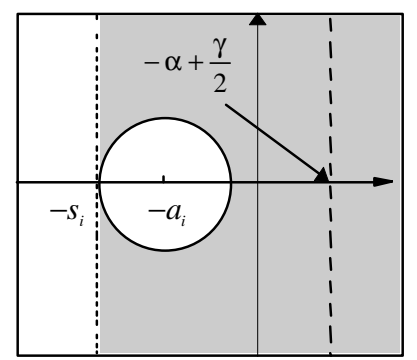

(b)
Figure 1. convergence domain of Laguerre series expansion

$$
\text { of } F(s)=\frac{1}{s+3} \text {. (a) } \alpha=2, \gamma=3 \text {, (b) } \alpha=2, \gamma=8
$$

\section{Remarks}

1. A convergence factor can also be defined which will give the rate of convergence of the geometrical series. Inequality (13) would then be replaced by $\frac{\overline{A S_{i}}}{\overline{B S_{i}}} \frac{\overline{B M}}{\overline{A M}}<f_{c}$. This implies that all $\delta_{i}$ 's must be replaced by $\delta_{i} / f_{c}$ in all the equations following (13).

2. The convergence condition may also be expressed using only the Laguerre parameters $\alpha$ and $\gamma$ together with the pole $s_{i}$ of $F(s)$. Indeed, it follows from (14):

$$
\begin{aligned}
& \delta_{i}^{2}-1=\frac{\gamma\left(-2 s_{i}+2 \alpha-\gamma\right)}{\left(-s_{i}+\alpha-\gamma\right)^{2}} \\
& r_{i}=\left|\frac{\left(s_{i}-\alpha\right)\left(s_{i}-\alpha+\gamma\right)}{2 s_{i}-2 \alpha+\gamma}\right| \\
& a_{i}=\frac{s_{i}^{2}-\alpha^{2}+\alpha \gamma}{2 s_{i}-2 \alpha+\gamma}
\end{aligned}
$$

Thus, it is possible to study the influence of the parameters $\alpha$ and $\gamma$ in the definition of the convergence domain. This study is carried out in the following section. 


\section{Discussion on the convergence domain}

According to (14a), the convergence domain is either inside or outside the circle defined by (15), depending on the quantity $\left(\delta_{i}^{2}-1\right)$.

The expressions (17a-c) can be studied in terms of $\gamma$, in order to represent the various convergence domains that can be obtained. The complete study will be omitted in order to alleviate this paper. Instead, the following three cases are going to be considered. They present a particular interest :

$$
\begin{aligned}
& \gamma_{1}=\alpha-s_{i} \\
& \gamma_{2}=2\left(\alpha-s_{i}\right) \\
& \gamma_{3}=\frac{\alpha^{2}-s_{i}^{2}}{\alpha}
\end{aligned}
$$

Indeed, $\gamma_{1}$ and $\gamma_{2}$ intervene in the study of the sign of $a_{i}$ (the abscissa of the center of the circle). The value $\gamma_{3}$ is useful to establish the sign of $\left(\delta_{i}^{2}-1\right)$ and hence whether the domain of convergence is inside or outside the circle. Finally, $\gamma_{1}, \gamma_{2}$ and $\gamma_{3}$ are used to compute the radius of the circle.

Among all the feasible situations, four of them are presented herein on the transfer function of example 1 (each situation has a particularity):

$$
F(s)=\frac{1}{s+3}
$$

For each example, the Fourier coefficients are computed and the domain of convergence, Nyquist diagram, step responses and responses to some random high frequency input signals are plotted for both the original transfer function and its approximation.

\section{Example 2}

The choice $\alpha=5, \quad \gamma=3.75$ yields the following characteristics of the convergence domain: $\delta_{i}=1.11, r_{i}=18$, $a_{i}=-15$. The first four Fourier coefficients are respectively: $2.143,-2.449,2.799$, and -3.199 .

Note that the Fourier coefficients are divergent. The convergence domain of the Laguerre series is located inside the circle of figure 5. Only the segment of the imaginary axis which is around the origin is included in the convergence domain. Thus, the Laguerre series is convergent only in low frequencies. This fact is validated by the Nyquist plot. In the time-domain, the step response can be judged satisfactory, the gain of the decomposition being correctly approximated (the real gain is 0.3333, the expansion gain is 0.3338). However, the response to a high frequency input signal is less satisfactory, as expected.

\section{Example 3}

The choice $\alpha=5, \gamma=6$ yields the following characteristics of the convergence domain : $\delta_{i}=0.5, r_{i}=4, a_{i}=-7$. The first four Fourier coefficients are respectively: $1.500,-0.750$ 0.351 and -0.187 . They are convergent now. In the time domain as well as the frequency domain the decomposition is judged satisfactory (the expansion gain is 0.3333). The convergence domain (figure 6), corresponds to the plane located on the RHS of the vertical axis at -3 . It extends the one obtained by the worst-case study (half plane at the RHS of the vertical axis at -2 ).

\section{Example 4}

The choice $\alpha=1.5, \quad \gamma=0.5$ yields the following characteristics of the convergence domain: $\delta_{i}=0.75$, $r_{i}=0.857, a_{i}=-2.143$. The first four Fourier coefficients are respectively: 0.2500 .1870 .141 and 0.105 .

The real gain remains unchanged, whereas the expansion gain equals 0.3125 . Here again the convergence domain was extended, compared to the worst case study (figure 7).

\section{Example 5}

The choice $\alpha=1.75, \quad \gamma=5$ yields the following characteristics of the convergence domain: $\delta_{i}=0.2$, $r_{i}=1.042, a_{i}=-1.958$. The first four Fourier coefficients are respectively: $-0.8,0.16,0.032$, and 0.0064 .

The coefficients converge to zero. Moreover, there is a good approximation in the Nyquist plane (figure 8) for all frequencies which is reflected by a good approximation of the responses to both signals (step and random). The worst case study does not include the imaginary axis in the convergence domain, contrary to the newly proposed approach.

\section{Extensions to the bilateral decomposition}

In this section signals defined for both positive and negative time domains are treated. The functions expansion principle on orthogonal basis holds again. The only difference is that both (positive and negative) parts of the signal are decomposed separately on Laguerre functions i.e. the parameters $\alpha$ and $\gamma$ change from one expansion to the other.

A non-causal signal $f(t)$ can always be written as an addition of the negative-time and the positive-time signals:

$$
f(t)=f^{-}(t)+f^{+}(t)
$$

where:

$$
\begin{aligned}
& f^{+}(t)= \begin{cases}0 & \text { if } t<0 \\
f(t) & \text { if } t>0\end{cases} \\
& f^{-}(t)= \begin{cases}f(t) & \text { if } t<0 \\
0 & \text { if } t>0\end{cases}
\end{aligned}
$$

It may be useful to consider the signal which is symmetrical to $f^{-}(t)$ in order to compute its Laplace transform. Hence, define:

$$
f^{-}(-t)= \begin{cases}0 & \text { if } t<0 \\ f(-t) & \text { if } t>0\end{cases}
$$

Using (5a), the Fourier coefficients of both expansions are given by:

$$
\begin{aligned}
& f_{n}^{+}=\int_{0}^{\infty} f^{+}(t) l_{n}(t) \gamma^{+} e^{-\left(\gamma^{+}-2 \alpha^{+}\right) t} d t \\
& f_{n}^{-}=\int_{0}^{\infty} f^{-}(-t) l_{n}(t) \gamma^{-} e^{-\left(\gamma^{-}-2 \alpha^{-}\right) t} d t
\end{aligned}
$$


Both poles $\alpha^{-}$and $\alpha^{+}$are chosen positive in order to obtain stable Laguerre filters used as expanding basis functions for the signals $f^{-}(-t)$ and $f^{+}(t)$ (the expansion is truncated at the order $N$ ). The Laplace transforms obtained from these expansions are :

$$
\begin{aligned}
& \tilde{F}^{+}(s)=\sum_{n=0}^{N} f_{n}^{+} \frac{\left(s-\gamma^{+}+\alpha^{+}\right)^{n}}{\left(s+\alpha^{+}\right)^{n+1}} \\
& \tilde{F}^{-}(s)=\sum_{n=0}^{N} f_{n}^{-} \frac{\left(s-\gamma^{-}+\alpha^{-}\right)^{n}}{\left(s+\alpha^{-}\right)^{n+1}}
\end{aligned}
$$

Hence, the bilateral Laguerre transform of $f(t)$ is

$$
F(s)=\tilde{F}^{-}(-s)+\tilde{F}^{+}(s)
$$

The bilateral expansion of a time signal on Laguerre functions can be summarized in the following steps:

decompose the positive-time part of $f(t)$, which gives $\tilde{F}^{+}(s)$,

decompose the positive-time part of $f(-t)$, which gives $\tilde{F}^{-}(s)$,

sum up both parts to obtain the desired expansion: $\widetilde{F}^{-}(-s)+\widetilde{F}^{+}(s)$.

Hence, the bilateral decomposition of the function:

$$
\begin{aligned}
& f^{+}(t)=\sum_{i=1}^{m_{1}} B_{i} e^{-b_{i} t} \\
& f^{-}(t)=\sum_{i=1}^{m_{2}} A_{i} e^{a_{i} t}
\end{aligned}
$$

is given by:

$$
\begin{aligned}
\tilde{F}(s) & =\sum_{n=0}^{N} \gamma^{+} \sum_{i=1}^{m_{1}} B_{i} \frac{\left(b_{i}-\alpha^{+}\right)^{n}}{\left(b_{i}-\alpha^{+}+\gamma^{+}\right)^{n+1}} \frac{\left(s-\gamma^{+}+\alpha^{+}\right)^{n}}{\left(s+\alpha^{+}\right)^{n+1}} \\
& -\sum_{n=0}^{N} \gamma^{-} \sum_{i=1}^{m_{2}} A_{i} \frac{\left(a_{i}-\alpha^{-}\right)^{n}}{\left(a_{i}-\alpha^{-}+\gamma^{-}\right)^{n+1}} \frac{\left(s+\gamma^{-}-\alpha^{-}\right)^{n}}{\left(s-\alpha^{-}\right)^{n+1}}
\end{aligned}
$$

and the convergence conditions (11) is now :

$$
\begin{aligned}
& \left|\frac{\left(b_{i}-\alpha^{+}\right)\left(s-\gamma^{+}+\alpha^{+}\right)}{\left(b_{i}-\alpha^{+}+\gamma^{+}\right)\left(s+\alpha^{+}\right)}\right|<1 \\
& \left|\frac{\left(a_{i}-\alpha^{-}\right)\left(s+\gamma^{-}-\alpha^{-}\right)}{\left(a_{i}-\alpha^{-}+\gamma^{-}\right)\left(s-\alpha^{-}\right)}\right|<1
\end{aligned}
$$

Consequently, applying the results of section 5, the convergence domain can be defined as:

$$
\Omega=\left\{\begin{array}{l}
x+j y,-\sup \left(b_{i}\right)<x<\inf \left(a_{i}\right), \\
\left(\left(\delta_{i}^{+}\right)^{2}-1\right)\left(\left(x+c_{i}^{+}\right)^{2}+y^{2}-\left(r_{i}^{+}\right)^{2}\right)<0 \\
\left(\left(\delta_{i}^{-}\right)^{2}-1\right)\left(\left(-x+c_{i}^{-}\right)^{2}+y^{2}-\left(r_{i}^{-}\right)^{2}\right)<0 \\
i=1, \ldots, m
\end{array}\right\}
$$

\section{Example 6}

When $m=1, a=1$ and $b=1$, the following definition results:

$$
f^{+}(t)=e^{-t} \quad \text { and } \quad f^{-}(t)=e^{t}
$$

Choosing $\alpha^{+}=2 / 3, \gamma^{+}=1 / 3$ and $\alpha^{-}=1 / 2, \gamma^{-}=1 / 3$, one gets : $\delta^{+}=1 / 2, \quad \delta^{-}=3 / 5, \quad c^{+}=7 / 9, \quad c^{-}=11 / 16, \quad r^{+}=2 / 9 \quad$ and $r^{-}$ $=5 / 16$.

The Fourier coefficients are given in the following table. Note that the positive and negative-time expansions are carried out respectively with 5 and 4 Laguerre functions.

\begin{tabular}{|l|l|l|}
\hline$n$ & $f_{n}^{+}$ & $f_{n}^{-}$ \\
\hline 1 & 0.400 & 0.500 \\
\hline 2 & 0.240 & 0.250 \\
\hline 3 & 0.144 & 0.125 \\
\hline 4 & 0.086 & 0.062 \\
\hline 5 & 0.052 & \\
\hline
\end{tabular}

Hence, the following decomposition can be written :

$$
\begin{aligned}
\tilde{F}(s)= & \frac{1}{s+2 / 3}\left(0.4+0.24 \frac{s+1 / 3}{s+2 / 3}+0.144\left(\frac{s+1 / 3}{s+2 / 3}\right)^{2}+\ldots\right) \\
& -\frac{1}{s-1 / 2}\left(0.5+0.25 \frac{s-1 / 6}{s-1 / 2}+0.125\left(\frac{s-1 / 6}{s-1 / 2}\right)^{2}+\ldots\right)
\end{aligned}
$$

Since the values of $\delta$ are less than 1, the convergence domain of $\tilde{F}(s)$ corresponds to the exterior of the circles defined previously.

Figure 2 shows the aspect of the convergence domain. It corresponds to the gray zone, comprised in the interval $-1<x<1$ and excludes the disks delimited by the circles:

$$
\begin{gathered}
(x+7 / 9)^{2}+y^{2}-(2 / 9)^{2}=0 \\
\text { and } \quad(x+11 / 16)^{2}+y^{2}-(5 / 16)^{2}=0 .
\end{gathered}
$$

The domain obtained by the worst-case study is restricted to the interval $-1 / 2<x<1 / 3$.

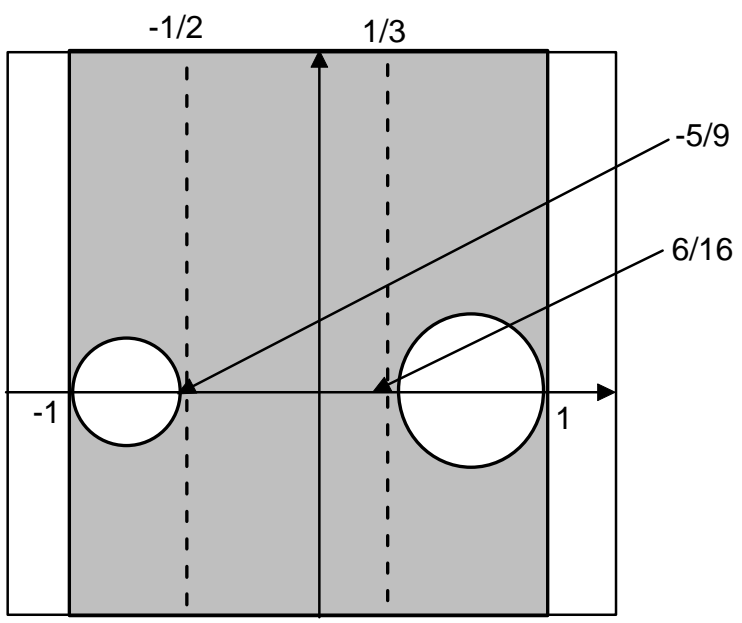

Figure 2. Convergence domain of the expansion of

$$
f(t)=\exp (-|t|)
$$


Figure 3 shows the superposition of the original signals and their expansions whereas figure 4 shows Bode plots in the interval $[0.1 \mathrm{rd} / \mathrm{sec} 10 \pi \mathrm{rd} / \mathrm{sec}]$.
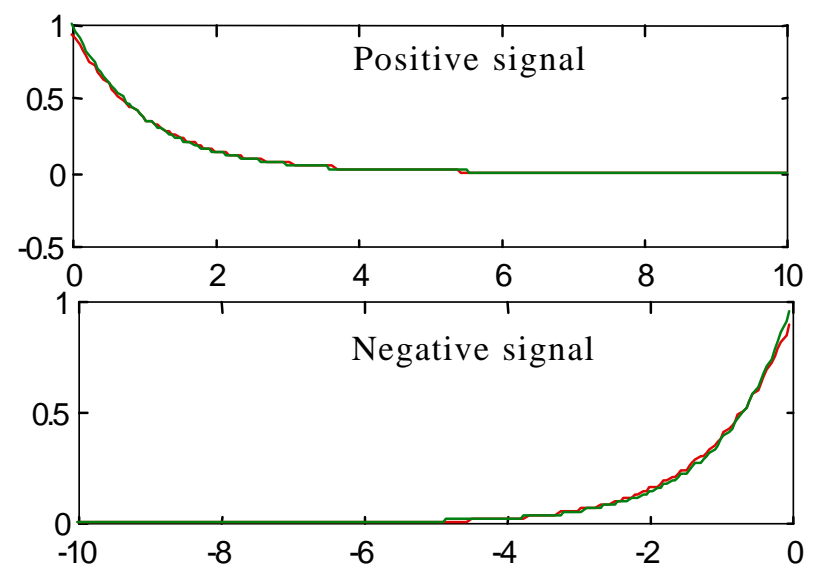

Figure 3. Bilateral expansion in the time domain

A simple example was selected on purpose for this paper. However, if more complex functions are treated, the computation of Fourier coefficients can be done using either numerical softwares such as Matlab or symbolic computation softwares such as Maple V or Mathematica.
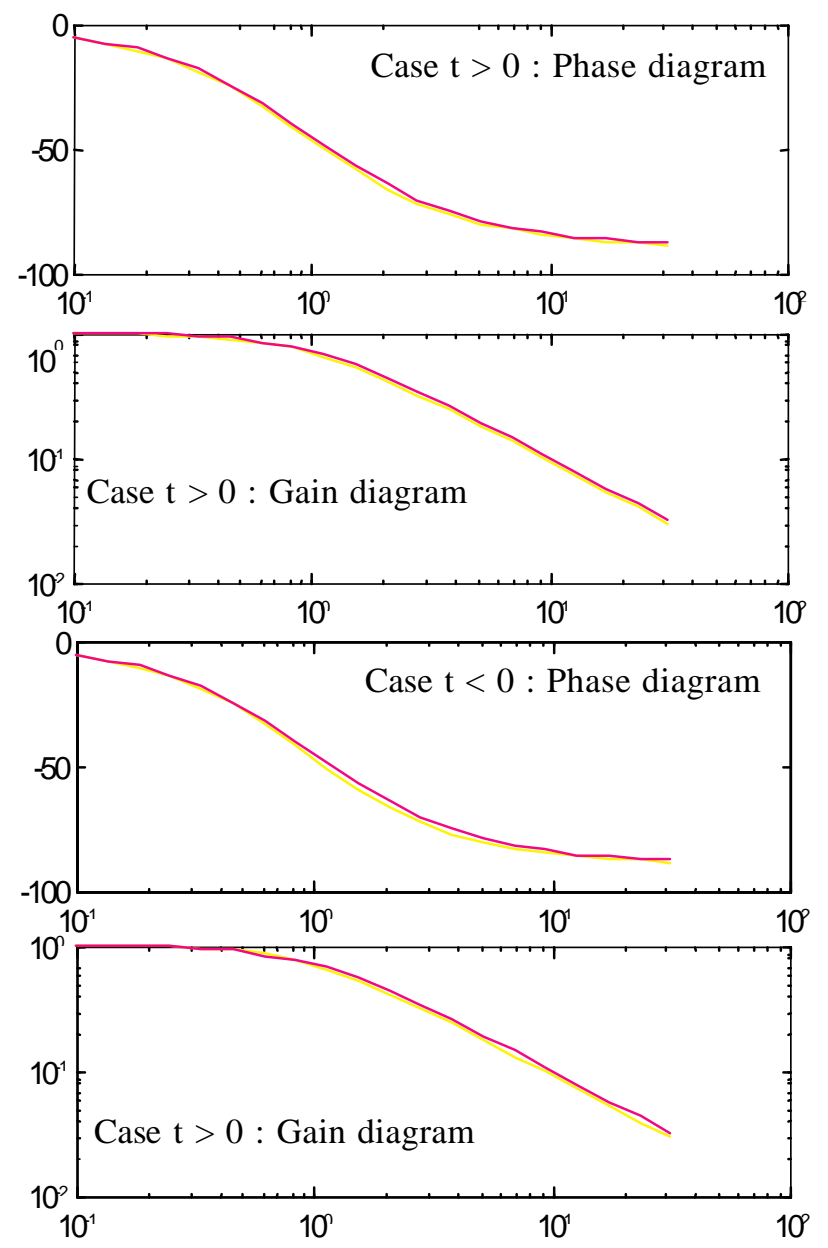

Figure 4. Bode plots

\section{Conclusion}

The convergence domain of the Laguerre series expansion was studied in this paper. From a theoretically point of view, the results obtained are important because they define the locus of the convergence domain in the complex $s$-plane.

In practice, it permits to the user to determine the frequency domain where the Laguerre model can correctly be utilized. As shown by different examples and by the analytical expressions, the tuning parameters $\alpha$ and $\gamma$ can be adapted to validate the approximation in a preferred frequency domain. The future developments of our work, concern the conception stage. Indeed, it would be interesting to propose an algorithm for choosing appropriate parameters to reach a convergence domain imposed by frequency constraints, for example. Moreover, the method presented for signals may be extended to the convolution relations in order to treat systems from input-output data.

\section{References}

Agamemnoni O., Paolini E., Desages A., On robust stability analysis of a control system using Laguerre series. Automatica, 28 (4), pp. 815-818, 1992.

Belanger R.P., Arafat O., Gaber M., Gendron S., VurgaitCherson D., Direct performance optimisation using Laguerre models. Automatica, 30 (5), pp. 883-996, 1994.

Kautz W.H., Transient synthesis in the time domain. Transactions Int. Radio Engrs., vol. CT1, pp. 29-39, 1954.

Malti R., Maquin D., Ragot J., Bilateral decomposition of a time function into Laguerre series. Application to LTI systems identification. Journal of Franklin Institute, vol. 335B, n 5, pp. 851-869, 1998a.

Malti R., Ekongolo S.B., Ragot J. Dynamic SISO and MISO system approximation based on optimal Laguerre models. IEEE Transactions on Automatic Control, 43 (9) , pp.13181323, 1998b.

Szegö G., Orthogonal polynomials, American Mathematical society. 1931

Wahlberg, B., System identification using Laguerre models. IEEE Transactions on Automatic Control, 36 (5), pp. 551$562,1991$.

Wang L., and Cluett W.R., Optimal choice of time-scaling factor for linear system approximations using Laguerre models. IEEE Transactions on Automatic Control, 39 (7), pp. 1463-1467, 1994. 

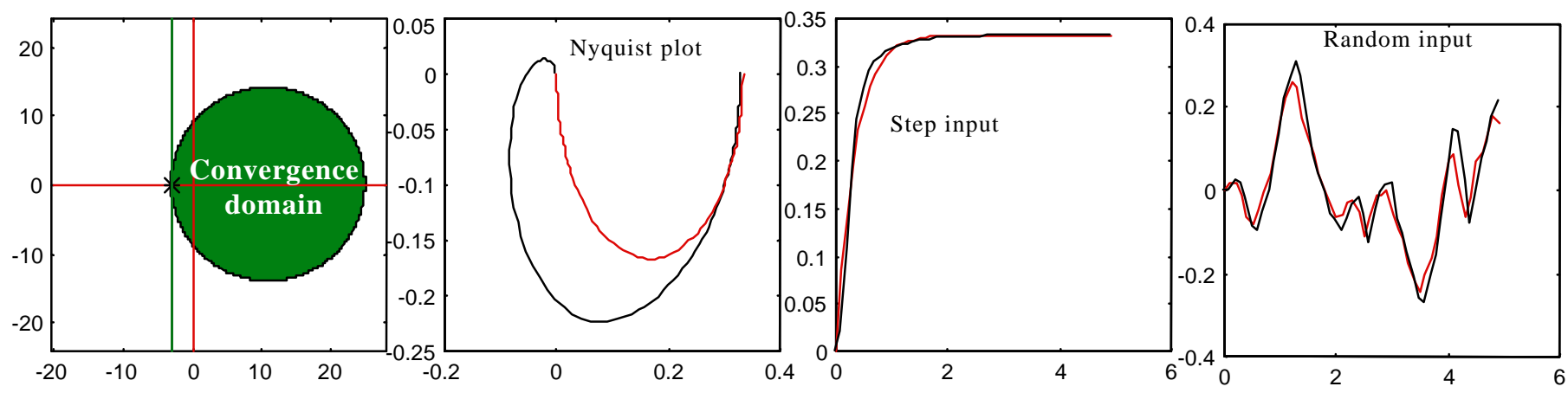

Figure 5. Expansion results of example 2
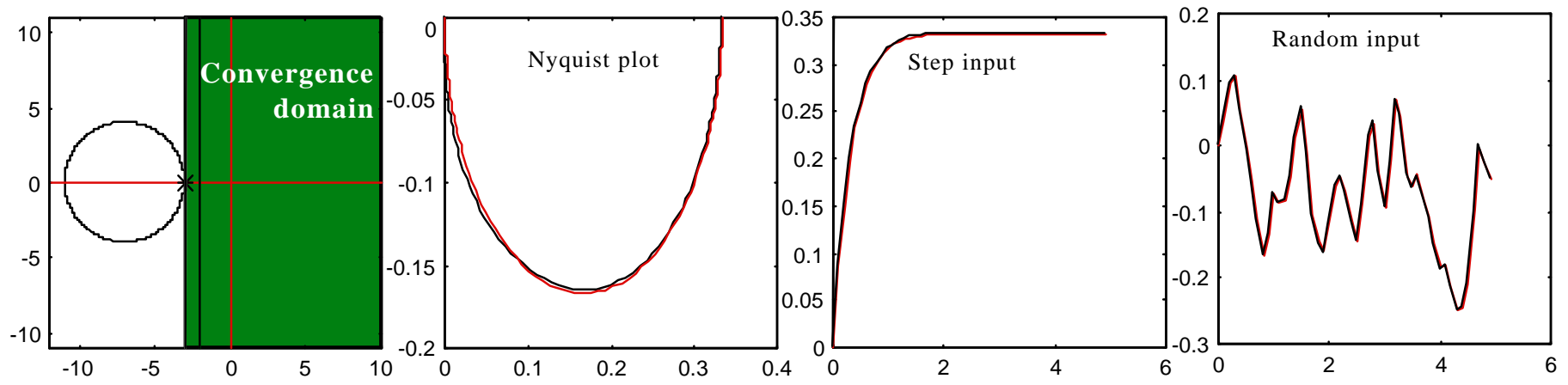

Figure 6. Expansion results of example 3
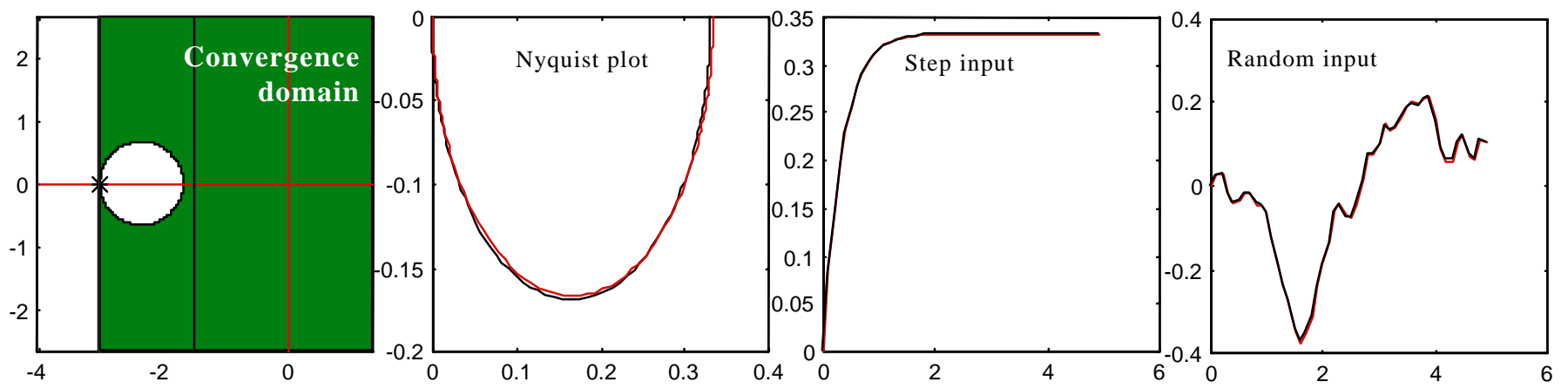

Figure 7. Expansion results of example 4
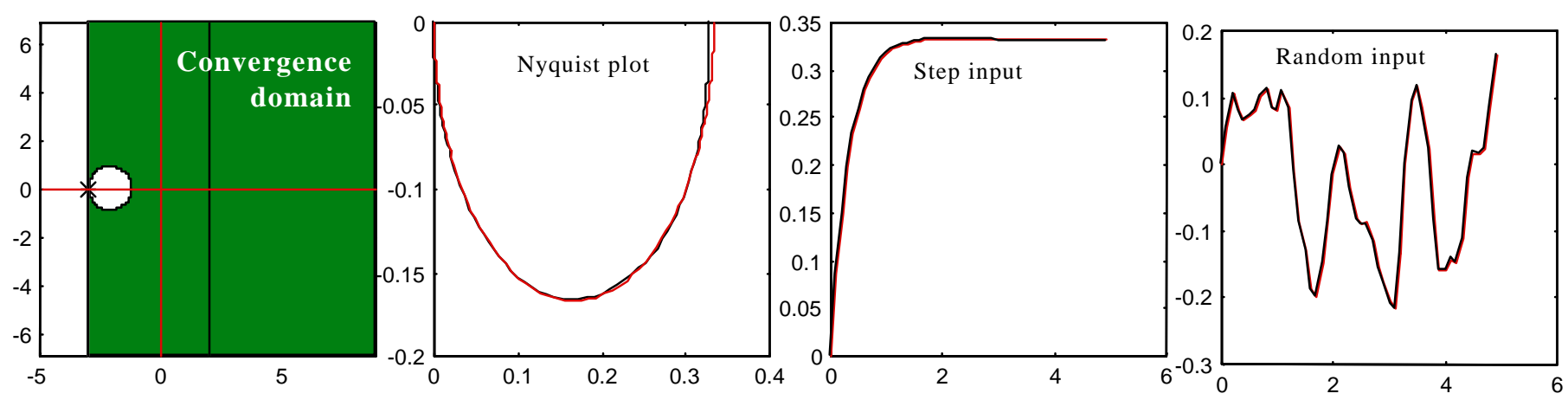

Figure 8. Expansion results of example 5 